\title{
MODELOS DE ATENCIÓN A LOS SÍNTOMAS SOMÁTICOS SIN CAUSA ORGÁNICA. DE LOS TRASTORNOS FISIOPATOLÓGICOS AL MALESTAR DE LAS MUJERES
}

\author{
Sara Velasco (1), Mª Teresa Ruiz (2) y Carlos Álvarez-Dardet (1, 2) \\ (1) Departamento de Salud Pública. Universidad de Alicante. \\ (2) Red de Género y Salud.
}

\section{RESUMEN}

Los síntomas somáticos sin causa orgánica (SSCO) permanecen médicamente inexplicados. Su abordaje podría estar limitado por un concepto biologicista de la salud. Diversas teorías críticas estudian factores contextuales, de género y subjetivos para los SSCO y sus modelos de atención. El objetivo de este trabajo ha sido revisar literatura española (1995-2002) explorando modelos explicativos y de atención a los SSCO relacionándolos con las teorías subyacentes sobre salud. Se realizó búsqueda en Medline, Sociologycal Abstracts, Psycinfo, Doyma, Google y Scirus, bajo palabras clave: Gender and health, Primary care, Somatic complaints and women or gender, Fibromialgy, Chronic fatigue, Functional syndromes, Feminist studies and primary care, malaise women. Seleccionando 31 artículos. Se clasificaron según teorías sobre salud: biomédica, psicosocial, socialista, biopsicosocial, ecosocial, psicoanalítica y feminista. Después, se identificaron modelos explicativos/de atención por bloques teóricos según: términos utilizados para SSCO; factores causales/asociados; modelos de atención; factores relación médicopaciente. En el marco biomédico se encuentran: explicaciones fisiopatológicas; atención directiva, centrada en la visión médica, tendente a adaptación del enfermo a su cronicidad con tratamientos sintomáticos y antidepresivos. En modelos teóricos críticos: relacionan síntomas con contexto social, experiencias subjetivas y de género; centrado en la visión de la/el paciente; evitan la medicalización, dirigido a construir el significado subjetivo de los síntomas, al empoderamiento de la mujer y su autorresponsabilidad. Conviene reconocer la existencia de teorías alternativas a la biomédica que amplían la comprensión de los SSCO, incluyendo factores contextuales, de género y subjetivos asociados al proceso de salud-enfermedad, que producen modelos de atención con mayor potencial de atención integral.

Palabras clave: Género. Salud de las mujeres. Feminismo. Psicoanálisis. Síntomas afectivos. Investigación biomédica. Modelos biológicos.
Correspondencia:

Sara Velasco Arias

C/ Sueca 35, 9

46006 Valencia

Correo electrónico: saravelasco@gmail.com

\section{ABSTRACT}

\section{Attention Models to Somatic Symptoms Without Organic Cause: From Phisiopathologic Disorders to Malaise of Women}

Somatic symptoms of no identifiable organic cause remain medically unexplained and have been delved into to only a limited degree from a biologicist conception of health. Different critical theories study contextual, gender-related and subjective factors for Somatic Symptoms of No Identifiable Organic Cause and their care models. This study has been aimed at reviewing published Spanish studies (1995-2002) exploring explanatory SSNIOC's by relating them to the underlying health theories. A search was run in Medline, Sociologycal Abstracts, Psycinfo, Doyma, Google and Scirus, using the key words: Gender and health, Primary care, Somatic complaints and women or gender, Fibromialgy, Chronic fatigue, Functional syndromes, Feminist studies and primary care, malaise women, a total of 31 articles having been selected and classified by health theories: biomedical, psychosocial, socialist, biopsychosocial, ecosocial, psychoanalytical and feminist.. Explanatory/care models were then identified by theory-related blocks, according to: terms use for SNIOC's; related/causal factors, care models, physician-patient relationship factors.

Within the biomedical framework, physicpathological, directive care explanations were found focusing on the medical viewpoint, tending toward adapting the patient to his/her degree of chronicity with symptomatic and antidepressant treatments. In critical theory models: symptoms are related to the social context, subjective gender experiences, focusing on the patient viewpoint; avoiding medicalization, aimed at constructing the subjective significance of the symptoms, on empowering the woman and on her taking responsibility for herself. It is advisable to recognize the existence of theories alternative to biomedical theory which broaden the comprehension of SSNIOC's, by including contextual, gender-related subjective factors related to the wellness-illness process which give rise to care models of greater integral care potential.

Key words: Gender. Women's health. Feminism. Psychoanalysis. Affective symptoms. Models, Theoretical. Biomedical Research. Models, Biological. 


\section{INTRODUCCIÓN}

Los síntomas somáticos sin causa orgánica (SSCO) incluyen una larga serie de quejas subjetivas para las que no se encuentran signos clínicos que las justifiquen. Puede tratarse de síntomas solos o agrupados (anexo 1) ${ }^{1}$. Algunos grupos de síntomas constituyen síndromes funcionales (anexo 2) que coexisten, a menudo, en los mismos pacientes y se suceden a lo largo de su vida. Los diagnósticos funcionales se solapan unos con otros ${ }^{2} y$ también con diagnósticos psicopatológicos como ansiedad y depresión ${ }^{3-5}$. El solapamiento puede depender, entre otros factores, tanto de que se apliquen clasificaciones nosológicas basadas en criterios para trastornos orgánicos que no se pueden objetivar en estos síntomas y síndromes, como de la consecuente interpretación subjetiva de los médicos. Los estudios de Nimnuan ${ }^{1}$, comprueban que pacientes con grupos similares de síntomas son diagnosticados de unos u otros síndromes funcionales dependiendo del especialista al que acudan: fibromialgia si acuden al reumatólogo; fatiga crónica si acuden al neurólogo y colon irritable si se trata del digestólogo. Cada especialista toma en consideración los síntomas que le son familiares y dejan fuera del diagnóstico una media de 8 síntomas que no encajan en su especialidad.

La prevalencia de los SSCO parece ser creciente $^{6-8}$ y es claramente más alta en mujeres ${ }^{9}$, de etiología mal conocida, de diagnóstico difícil y solapable ${ }^{1,2}$, de curso crónico, y no se cuenta con un tratamiento médico que se haya mostrado eficaz. Al menos utilizando los conceptos sobre salud y enfermedad de la teoría biomédica no se encuentran explicaciones consistentes para los hechos anteriores sobre los SSCO, ni se dispone de tratamientos fundamentados en factores etiológicos o asociados, o mecanismos de la enfermedad, sino sólo sintomáticos y paliativos. El panorama de la comprensión y abordaje terapéutico podría estar lastrado por los límites de la capacidad explica- tiva del concepto biologicista de la salud del paradigma biomédico.

Según un trabajo inédito de recopilación y caracterización de teorías de la salud realizado por los autores, existen teorías de la salud alternativas caracterizadas por diferentes conceptos del proceso de salud-enfermedad. Las teorías socialista ${ }^{10}$, biopsicosocial ${ }^{11-15}$, ecosocial $^{10,16}$ y psicoanalítica ${ }^{17,18}$, se caracterizan por incluir en el proceso salud enfermedad tanto el contexto social y la biografía como su interacción con la experiencia vivida y subjetiva de las personas. Ello implica que buscan etiología y factores causales o asociados en la biografía y la forma en que estos factores psicosociales son experimentados, es decir, el significado subjetivo. La teoría feminista, una teoría crítica más, añade los conceptos ligados a la diferencia sexual y la construcción social del género ${ }^{19-}$ 26 como factores del contexto socio-cultural que influye en el proceso de enfermar. Bajo estas perspectivas conceptuales se describe la influencia de factores del contexto social, de género y subjetivo sobre el fenómeno de los SSCO. Son factores que influyen: la clase social $^{8}$, el nivel cultural y la etnia ${ }^{27}$, la situación laboral, el estado civil ${ }^{8,28,29}$, y los estilos de vida y capacidad de respuesta a factores estresantes ${ }^{30}$ o eventos vitales. Se añaden factores de género: cuidado de hijos pequeños, carga del trabajo doméstico y múltiples roles ${ }^{28}$, feminidad y masculinidad y experiencia vivida ${ }^{31} \mathrm{y}$ análisis de género y poder y cómo este interviene en la presentación de los síntomas en las consultas, inmersas en un determinado tipo de relación médico-paciente ${ }^{32-34}$. Se trata de considerar múltiples factores psicosociales y sus interacciones ${ }^{35}$.

Bajo estas teorías deben producirse además de variaciones en los modelos explicativos, variaciones en los modelos de atención a los SSCO.

El objetivo del trabajo es revisar la literatura española del período 1995-2002, explo- 
rando modelos explicativos y de atención a los SSCO relacionándolos con las teorías subyacentes sobre la salud. Los objetivos instrumentales son 1. Clasificar los artículos revisados sobre síntomas somáticos sin causa orgánica según sus teorías subyacentes o explícitamente utilizadas. 2. Identificar los modelos de atención a los trastornos estudiados en base a los siguientes contenidos: términos que utilizan para los síntomas en estudio; factores causales y asociados que consideran; modelos de atención que proponen, factores que consideran en la relación médico-paciente.

\section{MATERIAL Y MÉTODO}

Se realiza una revisión de la literatura sobre síntomas somáticos sin causa orgánica. La búsqueda bibliográfica en castellano se restringió a los síntomas, quejas y síndromes sin causa orgánica presentados en atención primaria en mujeres y se ha realizado en las siguientes bases bibliográficas: MEDLINE, Sociologycal Abstracts, PsycINFO, DOYMA y buscador GOOGLE y SCIRUS, bajo las siguientes palabras clave:

Género y salud. Gender and health; Atención primaria y género. Primary care and gender; Somatización y género o mujeres. Somatic and gender or women; Quejas y mujeres o género y atención primaria. Complaints and women or gender and primary care; Fibromialgia. Fibromialgy síndrome; Fatiga crónica. Chronic fatigue síndrome; Síndromes funcionales. Functional sindroms; Estudios feministas y atención primaria. Feminist studies and primary care; Malestar de las mujeres. Discontent, malaise women.

Los criterios de selección, aplicados a través de los resúmenes, tratan de obtener artículos que muestren modelos explicativos o de atención relacionados con atención primaria.
Los criterios de inclusión han sido: artículos sobre SSCO relacionados con estudios de población general y atención primaria, salud mental, depresión, somatización, síndromes funcionales, dolor, dolores musculoesqueléticos, fatiga crónica, fibromialgia, trastornos mentales comunes, distress psicológico, países occidentales.

Los criterios de exclusión: artículos relacionados con el medio hospitalario, enfermedades orgánicas, pediatría, niños, adolescentes y tercera edad, enfermedades psiquiátricas mayores, trastornos de la alimentación, ginecología, reproducción y menopausia, medicinas alternativas, países del tercer mundo.

La búsqueda inicial bajo palabras clave género y salud (gender and health) permitió seleccionar fácilmente los artículos en bases de datos españolas aplicando los criterios de exclusión e inclusión, porque el número de registros encontrado era pequeño. Sin embargo, se amplió la búsqueda a bases internacionales y publicaciones específicas feministas, por que no se encontraron trabajos españoles de teorías contextuales y feministas. Se requirió limitar con palabras clave sucesivas (primary care and gender, Somatic and gender or women, Complaints and women or gender and primary care).

Se delimitó temporalmente el período de estudio a los años comprendidos entre 1995 a 2002, ambos incluidos. Los artículos se clasificaron en empíricos (referidos a una investigación de campo) y teóricos (revisiones, monográficos, editoriales). Se revisaron 2.740 registros y se seleccionaron mediante los resumenes 108 artículos. Entre ellos se seleccionaron para la revisión los 31 artículos teóricos encontrados sobre los síntomas somáticos sin causa orgánica ${ }^{36-66}$.

En la lectura de los artículos seleccionados se aplica una parrilla que los clasifica en sus correspondientes teorías, buscando en cada artículo la definición subyacente o 
explícita que utilizan para los cuatro conceptos fundamentales que caracterizan cada teoría: concepto de enfermedad, tipo de causalidad, modelo de atención de salud y factores en la relación médico-paciente. Se ha seguido la clasificación de teorías y conceptos definitorios realizada previamente en el trabajo «Teorías de la salud. Revisión de conceptos» (tabla 1).

Para los síntomas somáticos sin causa orgánica se extrae el marco explicativo y el modelo de práctica clínica identificando los siguientes contenidos: 1 . términos que utilizan para los síntomas en estudio; 2. factores causales y asociados; 3 . modelos de atención que proponen, 4 . factores que consideran en la relación médico-paciente (tabla 3 ).

\section{Clasificación de artículos sobre síntomas somáticos sin causa orgánica según marcos teóricos}

Los 31 sobre $\mathrm{SSCO}$ revisados (anexo 1) se han podido ubicar en once categorías, que se pueden agrupar en tres bloques teóricos. Se encuentran trabajos en los que se asocia la concepción biomédica, algunos asociada a la psico-social, que se incluyen dentro de un primer bloque que denominamos biomédi$\mathrm{co}^{36-44}$. No explicitan su marco teórico, por lo que es necesario deducirlo a partir de la identificación de los conceptos que utilizan (anexo 1).

Forman un segundo bloque -que denominamos contextual-subjetividad-, 6 artículos de los cuales 2 se basan en la teoría psicoanalítica ${ }^{45,46}, 3$ asocian criterios biomédicos y psicoanalíticos ${ }^{47-49}, 1$ asocia teoría biopsicosocial y psicoanalítica ${ }^{50}$. Prácticamente en todos ellos se explicita el marco teórico utilizado y en todos se pueden detectar explícitamente los criterios definitorios de su correspondiente marco.

Un tercer bloque denominado feminista agrupa 16 artículos que siempre combinan la teoría feminista con otra teoría; hay representación de artículos que combinan todas las teorías planteadas, tanto con biomédi$\mathrm{ca}^{51,52}$, como contextuales ${ }^{53-56}$ y de la subjetividad $^{57-66}$.

No se ha encontrado ningún trabajo que asocie el marco biomédico con teorías sobre el contexto social. No se han encontrado trabajos ubicables en las teorías socialista o ecosocial, a no ser que fueran también feministas.

\section{Modelos explicativos y de atención a los síntomas somáticos sin causa orgánica}

En los artículos revisados se identifican modelos de atención a síntomas somáticos sin causa orgánica que pueden resumirse en tres, -aunque con variaciones menores en cada trabajo-, correlacionados con los tres bloques de teorías clasificadas: el modelo biomédico-psicosocial, modelo biopsicosocial-psicoanalítico; y modelos feministas (tabla 2).

\section{Modelo biomédico y psicosocial}

Los términos definitorios para los síntomas y síndromes somáticos sin causa orgánica que utilizan los trabajos que se encuadran dentro del paradigma biomédico son siempre diagnósticos según las clasificaciones vigentes de enfermedades generales y mentales (CIE, Clasificación Internacional de enfermedades y DSM Diagnostical and Statistical Manual de la American Psychiatric Association), o trastornos fisiopatológicos psiquiátricos: «depresión y ansiedad, somatización o trastornos somatoformes» ${ }^{36,37}, \mathrm{y}$ síndromes funcionales de otras especialidades: «síndrome de fibromialgia, síndrome de fatiga crónica, síndrome de colon irritable, síndrome de piernas inquietas, disfunción temporomandibular, cefalea tensional» ${ }^{39,41}$. De manera accesoria se recogen términos 
Tabla 1

Resultados de ubicación de artículos revisados en teorías de la salud

\begin{tabular}{|c|c|}
\hline $\begin{array}{l}\text { Artículo. } \\
\text { Autor/a (nº cita) Año, País }\end{array}$ & Teoría explicitada o subyacente \\
\hline \multicolumn{2}{|c|}{ BLOQUE $1^{\circ}$. Paradigma biomédico y psicosocial } \\
\hline Gill, David (36) 1999 Reino Unido & Biomédico \\
\hline Ruiz Doblado, S (37) 1997 España & Biomédico \\
\hline Alijotas, Jaume (38) 2002 España & Biomédico \\
\hline Uceda, Julia (39) 2000 España & Biomédico \\
\hline Sanz-Carrillo, C, (40), 1998, España & Biomédico Psicosocial \\
\hline Moreno, Isabel (41) 2000 España & Biomédico Psicosocial \\
\hline Claraco Vega, LM (42) 1999 España & Biomédico Psicosocial \\
\hline Caballero Martínez, L. (43) 2002, España & Biomédico Psicosocial \\
\hline Arnetz, Bengt, B (44) 1996, Suecia & Psicosocial \\
\hline \multicolumn{2}{|c|}{ BLOQUE $2^{\circ}$. Contextual y subjetividad } \\
\hline García Campayo, J(45) 2000, España & Biomédico Psicoanalítico \\
\hline Iglesias Serrano, C(46) 2000, España & Biomédico Psicoanalítico \\
\hline López Herrero, LS (47)1997España & Psicoanalítico \\
\hline Aparicio, Daniela (48) 2002 España & Psicoanalítico \\
\hline de Luis Arribas, LM (48) 2000, España & Biomédico Psicoanalítico \\
\hline Tizón, José Luis (50) 2000, España & Biopsicosocial psicoanalítico \\
\hline \multicolumn{2}{|c|}{ BLOQUE $3^{\circ}$. Feminista } \\
\hline Richman, Judith A (51) 2001 & feminista-biomédica \\
\hline Valls Llobet, Carme(52) 2002 España & feminista-biomédica \\
\hline Stoppard, Janet M (53) 1987, Canadá & feminista-psicosocial \\
\hline OMS(54) 2002 Suiza & feminista-psicosocial \\
\hline Romito, Patricia (55) 1994, Italia & feminista-socialista \\
\hline Hankin, Janet R (56) 1990, EEUU & feminista-socialista \\
\hline Annandale, Ellen (57) 1990 G Bretaña & feminista-biopsicosocial \\
\hline Wool, Carol, A (58) 1994 EEUU & feminista- biopsicosocial \\
\hline Stewart, Donna E (59) 1996 Canada & feminista- biopsicosocial \\
\hline Gisjbers Van Wijk C MT (60) 1996, Holanda & feminista- biopsicosocial \\
\hline Busfield, Joan (61) 1988, EEUU & feminista- ecosocial \\
\hline Malterud, Kirsti (62) 1998, Noruega & feminista- ecosocial \\
\hline Malterud, Kirsti (63) 2000, Noruega & feminista- ecosocial \\
\hline Tubert, Silvia (64) 1999, España & feminista-psicoanalítica \\
\hline Bayo-Borrás, Regina (65) 2002, España & feminista-psicoanalítica \\
\hline Delgado, Ana (66) 2001 España & feminista-psicoanalítica \\
\hline
\end{tabular}


Resultados de modelos explicativos y de atención encontrados para síntomas somáticos sin causa orgánica agregados por bloques teóricos $(*)$

\begin{tabular}{|c|c|}
\hline \multicolumn{2}{|r|}{ Bloque biomédico-psicosocial } \\
\hline 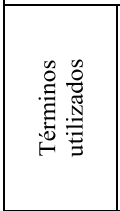 & $\begin{array}{l}\text { Trastornos psiquiátricos (DSM): somatización, manifestaciones somáticas de depresión: depresión enmascarada, } \\
\text { equivalente depresivo, depresión sin depression. } \\
\text { Pacientes somatizadores. Síndromes somáticos funcionales o psicógenos } \\
\text { Síndromes funcionales(CIE) : fatiga crónica, fibromialgia, intestino irritable, cefalea tensional, dismenorrea primaria, } \\
\text { s. miofascial,, migraña, disfunción temporomandibular, s. de piernas inquietas. Hiperfrecuentadores. Enfermedades } \\
\text { medioambientales. } \\
\text { Enfermedades del espectro afectivo. "Diagnósticos de moda". }\end{array}$ \\
\hline 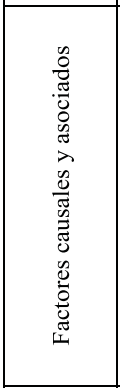 & $\begin{array}{l}\text { Etiología: Trastorno endógeno fisiopatológica ó exógena por estresores psicosociales que generan trastorno } \\
\text { fisiopatológico. } \\
\text { Biológicos no conocidos y estilos de vida. } \\
\text { Mala adaptación al estrés con factores ergonómicos y psicosociales. En los crónicos es "una forma de vida" } \\
\text { Hipótesis no comprobadas de alteración fisiopatológicas en la homeostasis por trastorno neuroendocrinoinmune, } \\
\text { disfunción corticohipofisosuprarrenal o vírica. } \\
\text { Factores asociados: enfermedad física o psiquiátrica y factores sociales estresantes biológicos: sexo femenino, falta de } \\
\text { forma física, alteraciones del sueño, alteraciones del mecanismo regulador del dolor, alteración del sistema de respuesta } \\
\text { al estrés. Cognitivas: cultura de dolor, hipervigilancia, estrategias de afrontamiento, autoeficacia percibida de control } \\
\text { del dolor, depresión ansiedad, rasgos de personalidad alterados, comportamientos frente al dolor. } \\
\text { Predisposiciones por influencia aprendida en la familia(carencias afectivas infantiles y experiencias repetidas de } \\
\text { enfermedad, predisposición familiar negativa hacia la enfermedad psiquiátrica); Insatisfacción laboral, conyugal y } \\
\text { social como estresores. Personalidad histriónica en mujeres y antisocial en hombres, o dependiente en ambos. }\end{array}$ \\
\hline 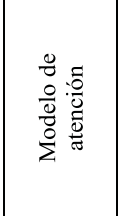 & $\begin{array}{l}\text { Diagnóstico por anamnesis sistemática de síntomas y criterios de conducta. Exploración y pruebas complementarias } \\
\text { para diagnóstico diferencial. Valoración psicopatológica por escalas autoadministradas. Clasificación CIE y DSM. } \\
\text { Tratamiento farmacológico sintomático del dolor y de soporte (antidepresivos, ansiolíticos, antiinflamatorios y } \\
\text { corticoides de efectos dudoso). Ejercicio progresivo controlado. Terapia cognitivo conductual para adaptación del } \\
\text { paciente. "Modelo de reatribución" para que el paciente atribuya sus síntomas a causa psicológica y acepte } \\
\text { farmacoterapia y psicoterapia. } \\
\text { Intervención familiar con modelo de reatribución y psicoeducativo }\end{array}$ \\
\hline \begin{tabular}{|lll}
0 \\
\hdashline
\end{tabular} & $\begin{array}{l}\text { Factores negativos: Desconcierto y frustración del médico por falta de daño orgánico objetivable y ausencia de } \\
\text { tratamiento eficaz, lo que deriva en falta de una conducta y actitud diagnóstica homogénea y rechazo de los pacientes } \\
\text { calificados de "dificiles", "simuladores" y "aprehensivos". } \\
\text { Factores positivos: capacidad y conocimientos técnicos }\end{array}$ \\
\hline \multicolumn{2}{|r|}{ Bloque contextual y subjetividad. Biopsicosocial psicoanalítico. } \\
\hline 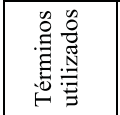 & $\begin{array}{l}\text { Quejas del cuerpo sin lesión corporal. Somatización.. Trastorno psíquico somatizado. Quejas biológicamente no } \\
\text { explicables } \\
\text { Lo psicosomático. "Malestar en la cultura, Histeria del s. XX". }\end{array}$ \\
\hline 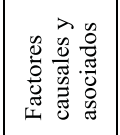 & $\begin{array}{l}\text { Etiología: Agentes externos, hábitos y conflictos intrapsíquicos. Conflicto personal inconsciente. } \\
\text { Factores asociados: Situaciones vitales de vulnerabilidad: pérdidas personales (muerte o separación, otras pérdidas). } \\
\text { Conflictos interpersonales (familia, trabajo), cambios en el desarrollo (adolescencia, jubilación). Inadaptación personal } \\
\text { (trabajo, hogar), cambios geográficos (emigración), estrés o aislamiento social. }\end{array}$ \\
\hline 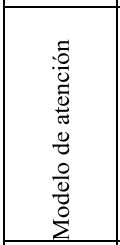 & $\begin{array}{l}\text { Diagnóstico descriptivo y comprensivo con integración interpretativa. Exploración biográfica y sucesos vitales. } \\
\text { Localización biográfica de los síntomas. Inducir comprensión de su malestar. Entrevista clínica y relación médico- } \\
\text { paciente prolongada centrada en el paciente y no en la enfermedad. como consultante además miembro de la } \\
\text { comunidad. Escucha psicoanalítica, significado simbólico de los síntomas, en consulta médica. Incluir al sujeto en la } \\
\text { relación médico-paciente y subjetivar el dolor. Psicoterapia grupal psicodinámica, cognitivo-conductual o } \\
\text { psicoeducativa. Apoyo teórico en "psicología clínica contemporánea" (cognitivo-conductual y psicoanalítico) } \\
\text { Atención integral, continuada en equipos interdisciplinarios y biopsicosociales, comunitaria, participativa, clínica } \\
\text { biopsicosocial con orientación psicológicamente influida }\end{array}$ \\
\hline 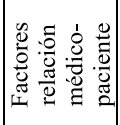 & $\begin{array}{l}\text { Factores negativos: la hiperdemanda e incapacidad de los pacientes para ser conscientes de su trastorno psicológico } \\
\text { provoca frustración, ira, rechazo contratransferencial y agresividad en los médicos por inadecuación del paciente. } \\
\text { Factores positivos: Conocimiento transferencial y comprensivo de la historia del paciente. Capacidad de escucha de lo } \\
\text { subjetivo e intervenir en situaciones familiares tras el paciente. }\end{array}$ \\
\hline
\end{tabular}


Tabla 2

Resultados de modelos explicativos y de atención encontrados para síntomas somáticos sin causa orgánica agregados por bloques teóricos (*) (Cont.)

\begin{tabular}{|c|c|}
\hline \multicolumn{2}{|r|}{ Bloque Feminista (biomédico-socialista-biopsicosocial-ecosocial-psicoanalítico) } \\
\hline 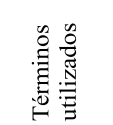 & $\begin{array}{l}\text { Problemas de salud mental, Enfermedad mental (DSM). Malestar o alteraciones psicológicas } \\
\text { Quejas somáticas. Síndromes funcionales } \\
\text { Síntomas sin enfermedad o síntomas de enfermedad no diagnosticada. Trastornos médicamente inexplicados. } \\
\text { Síntomas expresión del malestar de las mujeres. }\end{array}$ \\
\hline 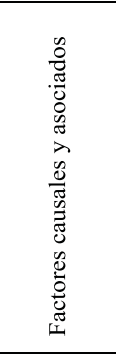 & $\begin{array}{l}\text { Etiología desconocida. Posible causa viral o neuroendocrinoinmune. E. Multicausales en interacción con el contexto } \\
\text { social y experiencia vivida, relacionada con género y poder. } \\
\text { Factores asociados: biológicos y psicosociales de género y su interiorización (biopsicosociales): Contexto social de } \\
\text { opresión de las mujeres: } \\
\text { Factores contingentes: Pobreza, dependencia económica, ser objeto de violencia, discriminación por sexo, raza, poder } \\
\text { limitado, discriminatoria calidad de los servicios sanitarios Relaciones de dominación social y familiar. Roles de } \\
\text { género desvalorizados, estereotipados: rol maternal, el conyugal, de ama de casa, cuidado de tres o más niños } \\
\text { pequeños, falta de apoyo de familia y amigos, falta de comunicación en la pareja. Modos de vida tradicionales, } \\
\text { Factores predisponentes: experiencias infantiles traumáticas. } \\
\text { Factores desencadenantes: violencia, migraciones, desarraigo, pérdidas de seres queridos. } \\
\text { Factores de protección: inserción laboral y social. Autonomía y control ante eventos severos; acceso a recursos } \\
\text { materiales, capacidad para elegir; soporte psicológico del entorno. }\end{array}$ \\
\hline 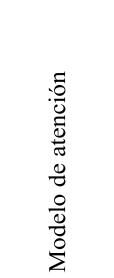 & $\begin{array}{l}\text { Biopsicosocial, centrado en la paciente y sensible al género } \\
\text { Redistribución del poder, reconociendo la experiencia de la paciente y retando la relación entre el poder de género y la } \\
\text { autoridad profesional. Estrategia de comunicación que invite a la paciente a usar su conocimiento en la vida diaria., } \\
\text { hacer emerger los significados de interacciones de género y poder como explicaciones significativas de los síntomas. } \\
\text { Grupos de autoayuda y discusión } \\
\text { Tratamiento biológico para el dolor y síntomas y ejercicio. Limitar psicofármacos, psiquiatrización y medicalización. } \\
\text { Terapias de orientación feminista: "taklking cure" y de autonomía, responsabilidad y socialización y empoderamiento } \\
\text { Favorecer la competencia de los/las sanitarias para reconocer y atender los factores de género } \\
\text { Promover la formulación e implementación de políticas de salud dirigidas a las necesidades de las mujeres. }\end{array}$ \\
\hline 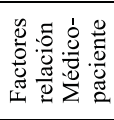 & $\begin{array}{l}\text { Factores negativos: Posibles sesgos de género que se manifiestan en errores diagnósticos. Efectos del poder médico, } \\
\text { como institución de saber y como institución masculina. Prejuicios e Intervención de patrones socioculturales } \\
\text { estereotipados de género por parte del médico/a } \\
\text { Factores positivos: capacidad para visualizar condicionantes de género y no funcionar con estereotipos }\end{array}$ \\
\hline
\end{tabular}

(*) Se presentan los resultados de modelos explicativos y de atención agregados por bloques teóricos y no por artículos y teorías porque, si bien la información no pierde coherencia, ésta sería excesivamente extensa. Sin embargo, dicha información está disponible para consulta contactando con las autoras/es.

como pacientes «simuladores, somatizadores hiperfrecuentadores» de servicios sanitarios, y se les califica de «pacientes difíciles, insatisfechos, hiperdemandantes, frustrantes para el médico» 36,37 .

En la teoría psicosocial se les llama «síndromes psicógenos, y enfermedades del espectro afectivo» y también «somatizadores» ${ }^{38-42}$.

Si los factores causales son desconocidos para el enfoque biomédico y las hipótesis expuestas como no demostradas, son de mecanismos fisiopatológicos que causen daño orgánico (neuro-endocrino-inmune o viral $)^{38,39}$, bajo la teoría psicosocial se apunta a factores causales psicológicos como respuesta al estrés. Estilos de vida, aprendizajes y creencias, y características como personalidad histriónica en mujeres, antisocial en hombres o dependiente en ambos se consideran predisponentes. Se señala que estos pacientes se niegan a reconocer o atribuir sus síntomas a causas psicológicas, y los trabajos refieren este punto como una fuente de frustración y desconcierto para los médicos que desemboca en el rechazo de los pacientes ${ }^{38.42}$. 


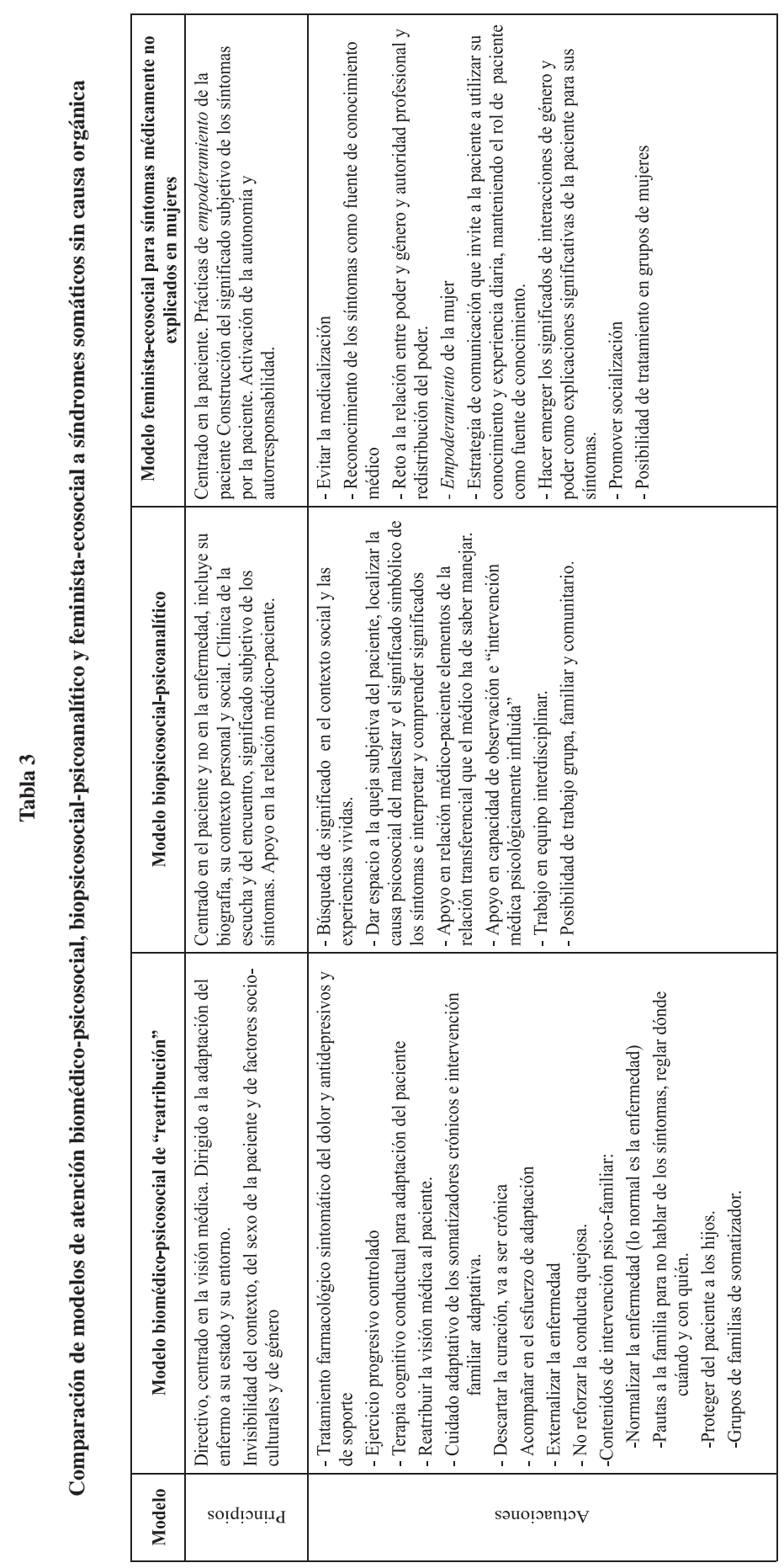


El modelo de atención biomédico se puede sintetizar como directivo, centrado en la visión médica, dirigido a la adaptación del enfermo a su estado y su entorno y no hace visibles el contexto, el sexo de la persona ni factores socio-culturales y de género. Se basa en la anamnesis de los síntomas, -que en el modelo psicosocial se completa con escalas autoadministradas de exploración psicopatológica-, seguidos de un diagnóstico sintomático que clasifica al paciente para desembocar en un tratamiento farmacológico de mantenimiento (analgésicos y antidepresivos). Las quejas médicas apuntan precisamente a las dificultades de clasificación de los pacientes porque los síntomas son numerosos, diversos, cambiantes, inespecíficos y solapados. Cuando se plantea el abordaje psicológico del paciente, que precisamente niega este aspecto, el objetivo de la intervención es «reatribuir» ${ }^{42}$ el padecimiento del paciente a su causa psicológica.

\section{Modelo psicoanalítico y biopsicosocial}

En los artículos revisados los términos describen no el síntoma que el médico explora sino las quejas, lo que el paciente siente, incluyendo así la escucha del sujeto. Añaden a los términos definitorios la ausencia de lesión orgánica: «quejas del cuerpo sin lesión corporal». Incluyen el mecanismo de conflicto psíquico que se expresa somáticamente: lo «psicosomático o trastorno psíquico somatizado». Encuadran los trastornos estudiados como un equivalente de la histeria del siglo XIX, en la medida en que lo consideran producto del malestar en la cultu$\mathrm{ra}^{46-48}$.

Algunos trabajos incorporan la influencia de la dinámica del conflicto psíquico, pero manteniéndose dentro de la psiquiatría descriptiva y clasificatoria, estos son mixtos biomédico-psicoanalíticos, que continúan llamando a los pacientes somatizadores o con síntomas físicos inespecíficos ${ }^{47}$. Cuando la teoría psicoanalítica se asocia con la teoría biopsicosocial la definición se fija en las quejas (componente subjetivo), biológicamente no explicadas, definiéndose los trastornos como «la expresión del malestar personal y social en un idioma de quejas corporales con búsqueda de ayuda médica» ${ }^{50}$.

En cuanto a los factores causales o asociados, las teorías psicoanalítica y biopsicosocial apuntan a los conflictos inconscientes frente a situaciones de vulnerabilidad como pérdidas, conflictos interpersonales, relaciones familiares, cambios en el desarrollo y situaciones sociales. Los síntomas se consideran efectos del contexto en el psiquismo y el cuerpo.

El modelo de atención se centra en el paciente y el conocimiento sobre sí mismo, incluyendo su biografía, su contexto personal y social. En consecuencia las intervenciones se apoyan en la escucha en entrevista clínica, dar espacio a la queja subjetiva del paciente, localizar la comprensión del malestar y el significado simbólico de los síntomas. Apoyo crucial en la relación médico-paciente, dinámica de la clínica del encuentro $^{47-50}$.

El modelo biopsicosocial incluye atención al contexto social y trabajo en equipo interdisciplinar. Si bien consideran en la relación médico-paciente la frustración del médico y la hiperdemanda e incapacidad de los pacientes para ser conscientes de su trastorno, estas dificultades se enfocan como elementos de la relación transferencial que el médico ha de saber manejar apoyándose en su capacidad de observación y de «intervención médica psicológicamente influida ${ }^{50}$ (tabla 2). La atención psicoanalítica no hace referencia a tratamientos farmacológicos.

\section{Modelo feminista}

Todos los artículos bajo teoría feminista diferencian el sexo de los y las pacientes y de 
los y las médicas, lo cual interviene en los padecimientos. Buscan explicación a la mayor prevalencia de SSCO en mujeres y su peor evolución, teniendo en cuenta que esos hechos han de tener un significado relacionado con la condición social y subjetiva de las mujeres ${ }^{58}$.

Los artículos feministas-biomédicos conservan los términos de síndromes funcionales a la par que tienden a rechazar los términos psiquiatrizantes de los síntomas, especialmente la antigua neurastenia ${ }^{51,52}$. Las categorías feministas hablan sistemáticamente de quejas y malestar: «quejas somáticas, malestar psicológico, síntomas del malestar», en el sentido psicoanalítico del malestar en la cultura. Se define el malestar de las mujeres como «una sensación subjetiva de padecimiento psíquico que no se encuentra dentro de los criterios clásicos de enfermedad, sino que se corresponde con conflictos psicosociales ${ }^{24}$. «Es el sufrimiento que no puede ser descifrado y expresado en palabras y que aparece en el cuerpo como síntomas mal definidos y sin causa orgánica demostrable».Además, especialmente en el feminismo-ecosocial, se definen como «síntomas médicamente no explicados, síntomas sin enfermedad o síntomas de enfermedad no diagnosticada» ${ }^{61,62}$.

La observación de factores causales, asociados, predisponentes y también protectores se basan en un núcleo central explicativo que es la opresión de las mujeres por su condición social subordinada, analizando las diferentes formas en que se manifiesta su deprivación de poder social y familiar. El feminismo-biomédico añade a los factores estresantes generales, la violencia, el abuso continuado y los abusos infantiles en la historia personal de las mujeres con síndromes somáticos $^{51}$. El feminismo-socialista destaca el contexto histórico, y socioeconómico en que viven las mujeres, incorporando los factores sociales de género: la situación marital, múltiples roles, ser madre de niños pequeños y el trabajo asalariado ${ }^{55,56}$.
El feminismo-psicosocial añade a los posibles factores estresantes generales los mismos factores sociales de género anteriores, detectando además factores protectores de género como el empoderamiento de las mujeres a través de la autonomía y el control de eventos vitales, acceso a recursos materiales y apoyo y soporte del entorno ${ }^{53,54}$. El feminismo-biopsicosocial, ecosocial y psicoanalítico se diferencian del socialista y psicosocial en que añaden a la fenomenología social de género, la importancia de la experiencia vivida. Aparecen nuevos factores relacionados con los significados subjetivos, como coherencia con los ideales de feminidad y masculinidad, resiliencia, control autopercibido ${ }^{57-63}$. Por último, lo específico del feminismo-psicoanalítico son los factores que dependen de la construcción de la subjetividad bajo las influencias de los consenso sociales de género, relaciones de dominación social y familiar, la desvalorización de los roles femeninos y conflictos de identidad, las pérdidas y el aislamiento ${ }^{64,66,67}$. En el anexo 3 se relacionan los factores asociados que son el cuerpo de investigación de los estudios feministas.

El modelo de terapia de orientación feminista se basa siempre en influir en los factores de opresión de género, familiar y social. Se sintetiza como centrado en la paciente. Prácticas de empoderamiento de la paciente y construcción del significado subjetivo de los síntomas por la paciente con activación de la autonomía y autorresponsabilidad. Desde los trabajos que se han ubicado en el feminismo ecosocial, categoría que contiene y sintetiza los conceptos de las anteriores, se apoyan en un modelo general de salud de las mujeres basado en un «trabajo consciente de dar cuidado a mujeres visualizando los problemas relacionados con su socialización y su posición como mujeres en esta sociedad, y ayudarlas a desarrollar estrategias para conseguir más autoridad y responsabilidad sobre sus propios cuerpos y sus vidas. Se sintetiza en: 1) consideración de la identidad de género de la paciente y de los roles de 
género; 2) Consideración de la situación personal y social de la paciente; 3) Trato respetuoso; 4) encaminar a la paciente a enfrentarse con sus problemas de salud y estimular su autorresponsabilidad; 5) evitar la medicalización». Este modelo general de atención a la salud de las mujeres, cuya estrategia para los SSCO se basa en: «empoderamiento de la mujer redistribuyendo el poder, reconociendo la experiencia de la paciente, retando a la relación entre poder y género y autoridad profesional. Estrategia de comunicación que invite a la paciente a utilizar su conocimiento y experiencia diaria, manteniendo el rol de la paciente como fuente de conocimiento. Hacer emerger los significados de interacciones de género y poder como explicaciones significativas de la paciente para sus síntomas. Posibilidad de tratamiento en grupos de mujeres» ${ }^{63}$.

En la relación médico-paciente las teorías feministas analizan la interacción de los estereotipos de género y su efecto sobre las actuaciones diagnósticas y terapéuticas de los médicos y médicas.

En la tabla 4 se sintetizan los tres modelos principales encontrados.

\section{COMENTARIOS}

Los modelos explicativos y de atención a SSCO identificados se pueden reducir a tres: el biomédico-psicosocial y los dos alternativos producidos bajo teorías críticas: el biopsicosocial-psicoanalítico y el feminista que se apoyan en concepciones diferentes del funcionamiento humano y de la salud.

Los síntomas y síndromes somáticos parecen situarse en la encrucijada entre el cuerpo, la mente y lo social, y los modelos dependen de cuáles de estas esferas se contemplen, limitando o ampliando su capacidad de comprensión del fenómeno.

Podemos identificar una transición conceptual entre los modelos que va desde defi- nir los síntomas somáticos como «trastornos fisiopatológicos» (psiquiátricos o funcionales), hasta «síntomas del malestar cultural de las mujeres»

En el modelo biomédico-psicosocial, el cuerpo es el objeto de la mirada médica que describe lo objetivable-signos, analíticas y escalas psicopatológicas en las que la mente es tomada también como un órgano- para llegar a un diagnóstico descriptivo de la enfermedad y no del significado del padecimiento para el paciente. El problema es que las evidencias apuntan a que los sistemas de clasificación diagnóstica basados en síntomas y escalas son escasamente válidos para estos trastornos ${ }^{1} 230$.Una posible consecuencia de ello es la calificación de estos pacientes, en los trabajos biomédicos, como inadecuados, de los que se desconfía y nombrarlos, no por la enfermedad que padecen, -por ejemplo, enfermedad por somatización-, sino por lo que hacen -paciente simulador o somatizador-, depositando un carácter de intencionalidad en el paciente, o bien, definirlos por su actitud o conducta,-hiperfrecuentadores, aprensivos, irritables, difíciles.

El modelo de atención biomédico se restringe a un hipotético funcionamiento fisiopatológico que, no por desconocido e improbado, deja de tomarse como causa. Se aplican los tratamientos farmacológicos disponibles (psicofármacos y analgésicos), aún con evidencias reconocidas de escasa eficacia y bajo la convicción de que los trastornos por somatización en realidad no tienen cura, al menos biológica. Se aplican actuaciones directivas, se intenta conseguir que el paciente «reatribuya»su padecimiento a la versión médica, tratándolos como «enfermos sin enfermedad» o que ésta es psicológicamente auto-causada, y que se adapte a ella; que padecen un error de funcionamiento, tratando de convencerle de que no se va a curar y que ha de adaptarse a su sufrimiento crónico con intervenciones que pauten cuándo, cómo y con quién debe el paciente mani- 
festar su dolor. Hay tensión entre lo que el médico espera del paciente, al que encuentra inadecuado, frustrante y difícil, y lo que el paciente espera de la medicina, que no alivia su sufrimiento. El encuentro en esta tensión está marcado por una cierta lucha de poder y rechazo mutuo $3^{3,32}$.

Desde la categoría psicoanalítica se discute la pertinencia de los términos biomédicos calificatorios de los y las pacientes por sus acciones y proponen nombrar los padecimientos, -trastorno psíquico somatizado-, incluyendo así además la hipótesis causal que es el conflicto psíquico. Para este segundo modelo biopsicosocial-psicoanalítico, la persona está en la historia, tiene biografía, y lo que le ocurre y sus síntomas tienen significados. Ya no se trata sólo de qué le pasa a la o el paciente, sino porqué le pasa insertado en su biografía. No es tan importante describir, clasificar y diagnosticar, sino interpretar y comprender significados. Apoyan su centro de gravedad en la comprensión del funcionamiento humano como una interacción entre la situación social y la subjetividad. El giro fundamental con respecto a los biomédicos está en que se buscan los factores causales o asociados en el contexto social y las experiencias vividas.

Se desplaza la mirada sobre el o la paciente como sujeto, escuchando sus quejas y se propone una clínica de la escucha, centrada en el paciente (tabla 4).

Las teorías feministas, -segundo bloque crítico-, además de incluir el contexto y la subjetividad añaden una aportación principal, y es que hacen aparecer la diferencia sexual como fuente de comprensión del fenómeno. El hecho de que la mayor parte de los pacientes de que hablamos sean mujeres permanece opaco en las anteriores teorías, sin embargo, el sexo puede contribuir a determinar y explicar los fenómenos tanto para los y las pacientes como de las actuaciones de médicos y médicas. Se evidencia que los estereotipos de género pueden actuar como determinantes de diferencias por $\operatorname{sexos}^{9,22,26,29}$.

El malestar de las mujeres, -concepto procedente de la teoría feminista ${ }^{24} 64,69$-,es producto de su posición social y de la vivencia de sus experiencias, y se presenta como una línea explicativa alternativa de los síntomas somáticos sin causa orgánica, producto de la asociación de las teorías feminista, contextuales y de la subjetividad, que incluye una concepción culturalista del funcionamiento humano y de la enfermedad. Esta perspectiva abre enigmas de investigación nuevos sobre el malestar de las mujeres que componen el cuerpo de investigación feminista que incorporan factores asociados y protectores contextuales, subjetivos y de género ${ }^{59,60,62,66,67}$ que se multiplican al indagar en los factores que gravitan sobre las formas de vida de las mujeres (tabla 3). Las propuestas terapéuticas se relacionan con la modificación de los factores de opresión de las mujeres, la recuperación de la autonomía y auto-responsabilidad. El modelo pivota sobre la devolución de la palabra a las mujeres.

Una de las limitaciones de este trabajo es que la clasificación de teorías realizada no excluye que existan otras teorías no recogidas y que sea susceptible de mayor complejidad o de otros límites basados en otros conceptos no considerados. Otra limitación procede de que se ha revisado una muestra limitada de artículos ya que no se ha hecho inclusión exhaustiva sino saturación de trabajos para cada marco teórico y que aplicando otros criterios de selección, o teniendo en cuenta artículos empíricos que no se han considerado, pudieran aparecer más modelos o variantes de los encontrados.

En cuanto a implicaciones de los resultados del presente trabajo podemos considerar la conveniencia de revisar y discutir los marcos teóricos que subyacen a los modelos de atención que se utilizan, ya que pueden estar limitados por concepciones parciales 
del proceso salud-enfermedad. Cuando se actúa bajo protocolos aparentemente sólo técnicos, los médicos y médicas están actuando siempre bajo concepciones teóricas determinantes y bien definidas del funcionamiento humano y de la salud, aunque no estén explicitadas en su formación, pero que dirigen sus pautas diagnósticas y terapéuticas. En el caso de los padecimientos por SSCO, muy ligados a factores contextuales, de género y subjetivos, desde concepciones biomédicas, puede estar dejándose fuera precisamente los factores asociados y modelos de atención que podrían ser más eficaces.

\section{CONCLUSIONES}

El abordaje de los SSCO desde el paradigma biomédico se presenta limitado porque su teoría de la salud no incluye los aspectos del funcionamiento humano que probablemente determinan el problema. Es conveniente reconocer la existencia de teorías alternativas a la biomédica que amplían las posibilidades de comprensión del problema, incluyendo factores contextuales, de género y subjetivos asociados al proceso de saludenfermedad y que producen modelos de atención que incluyen dichos aspectos, con mayor potencial de atención integral a los y las pacientes con SSCO.

\section{Anexo 1}

Listado de síndromes somáticos funcionales ${ }^{1}$

Dificultad para mantener el sueño

Despertar temprano

Dormir demasiado poco

Angustia al caer dormido

Fonosensibilidad

Fotosensibilidad

Palpitaciones

Temblor

Vértigo

Cambios de humor

Pesadillas

Rigidez matutina

Dolor persistente

Dolor lumbar

Sentir dolor por todas partes

Entumecimiento

Dolor pélvico

Reacciones a químicos o alimentos

Dolor facial
Dolor torácico

Dificultad para respirar

Ardor

Molestia en la garganta

Dolor abdominal

Cansancio mental

Cansancio físico

Somnolencia diurna

Irritabilidad

Fallos de memoria

Boca seca

Alteración del gusto

Zumbidos

Síntomas menstruales

Nauseas

Vómitos

Picor

Cefalea 


\section{Anexo 2}

Listado de síndromes somáticos funcionales (Nimnuan, 2001) (1)

Síndrome de colon irritable

Disfunción articular temporomandibular

Dolor facial atípico

Dispepsia no ulcerosa

Dolor pélvico crónico

Cefalea tensional

Fibromialgia

Síndrome de fatiga crónica

Bolo histérico

Sensibilización química múltiple

Hiperventilación

Síndrome premenstrual

Dolor torácico no cardiaco

\section{Anexo 3}

Cuerpo de investigación feminista en estudios de género sobre factores predisponentes en las mujeres a síntomas somáticos médicamente no explicados.

Constelación factorial predisponente: opresión de las mujeres y dominación social y familiar

Factores sociales de género:

El matrimonio

El trabajo de ama de casa

La doble jornada laboral o roles múltiples

Discriminación laboral

Tener tres o más niños pequeños

La falta de soporte o ayuda de amigos o familiares

La falta de comunicación íntima y confidencial con la pareja

Factores de significados subjetivos en experiencia vivida:

Experiencia infantiles traumáticas

Abusos infantiles

Violencia

Abuso continuado

Migraciones

Desarraigo, desinserción social y aislamiento

Pérdidas y muerte de seres queridos

Desvalorización de los roles femeninos

Ideales de feminidad y masculinidad

Características psicológicas estereotipadas de género:

Abnegación

Ser complaciente

No asertividad

Cuidadora

Dependencia 


\section{BIBLIOGRAFÍA}

1. Nimnuan $\mathrm{CH}$, Rabe-Hesketh S, Wesseley S, Hotopf M. How many functional somatic syndromes? J Psychosom Res 2001;51(4):549-57.

2. Wessely S, Nimnuan C, Sharpe M. Functional somatic syndromes, one or many. Lancet 1999; 354: 936-9.

3. Henningsen P, Jakobsen T, Schiltenwolf M, Weiss MG. Somatization Revisited: Diagnosis and Perceived Causes of Common Mental Disorders. Journal of Nervous \& Mental Disease 2005;193(2):85-92.

4. Fink P, Sørensen L, EngbergM, Holm M, MunkJørgensen. Prevalence, Health Care Utilization, and General Practitioner. Recognition Somatization in Primary Care. Psychosomatics August 1999; 40:330-8.

5. Lewis G, Wessely $\mathrm{S}$. The epidemiology of fatigue: more questions than answers. J Epidemiol Community Health 1992;46:92-7.

6. Organización Mundial de la Salud. Carga de los trastornos mentales y conductuales. En: Informe de salud en el Mundo 2001. Salud mental: nuevos conocimientos, nuevas esperanzas. [citado $10 \mathrm{de}$ nov. 2003]. Disponible en: www.who.org.

7. Organización Mundial de la Salud. Gender and women`s mental health. 2001 [citado 10 de nov. 2003]. Disponible en: www5.who.int/mental_ health.

8. Fahrenberg J. Somatic complaints in the German population. J Psychosom Research. 1995 Oct; 39(7): 809-17.

9. Kroenke K, Spitzer RL. Gender differences in the reporting of physical and somatoform symptoms. Psychosom Med 1998 Mar-Apr; 60 (2): 150-5.

10. Krieger N. Theories for social epidemiology in the 21st century: an ecosocial perspective. Int J Epidemiol 2001;30:668-77.

11. DubosR. El espejismo de la Salud. Utopías, progreso y cambio biológico. México: Fondo de Cultura Económica; 1975 (e. o. 1959).

12. Engel G. The need for a new medical model: a challenge for biomedicine. Scien 1977;196:129-36.

13. Cassel J. The contribution of the social environment to host resistance. Am J Epedemiol 1976; 104:107-23.
14. Borrel i Carrió F. El modelo biopsicosocial en evolución. Med Clin (Barc) 2002;119(5):175-9.

15. Porta M, Álvarez-Dardet C, Fernández E. Presentación. Epidemiología, ecología y epistemología: nuevas prácticas y paradigmas para la salud pública en la aldea global. Rev Salud Pública; 5:14-21.

16. Krieger N. Epidemilogy and the web of causation: has anyone seen the spider? Soc Sci Med 1994; 39 : 887-903.

17. Freud S. Obras completas. Madrid: Biblioteca Nueva; 1972.

18. Tizón García J. Los nuevos modelos asistenciales en medicina: una visión a partir de algunos fundamentos psicológicos y epistemológicos. Revisiones en Salud Pública 1995;4:57-83.

19. Mollet K. Política sexual. Madrid: Cátedra; 1995. 1969.

20. Marecek J, Kravetz D. Mujer y salud mental : un análisis de los intentos feministas de cambio. (e. o. 1973). En: Sáez Buenaventura C.(comp). Mujer, locura y feminismo. Madrid: Dédalo; 1979.

21. Fox Keller E. Reflexiones sobre género y ciencia. Valencia: Alfons el Magnanim; 1991.

22. Borges S; Waitzkin H. Women's narratives in primary care medical encounters. Women-Health 1995; 23(1): 29-56.

23. Rubin G. «The traffic in women: Notes on the 'Political Economy' of sex». En: Reiter, Rayna (comp.): Toward an Anthropology of Women, Nueva York y Londres, Monthly Review Press; 1975.

24. Burin M. El malestar de las mujeres. La tranquilidad recetada. Buenos Aires: Paidós; 1990.

25. Fee E. Woman and Health Care. The politics of sex in medicine. New York, 1983.

26. Ruiz MT. Igualdad de oportunidades en los servicios sanitarios: sesgo de género como determinante de la estructura de salud de la Comunidad. En: Miqueo, Consuelo (ed) Perspectivas de género en salud. Fundamentos científicos y socioprofesionales de diferencias sexuales no previstas. Madrid: Minerva; 2001.

27. Campbell JL, Ramsay J, Green J. Age, gender, socioeconomic, and ethnic differences in patients' assessments of primary health care. Qual Health Care 2001 Jun; 10(2): 90-5. 
28. Arber S. Class, Paid Employment and Family Roles: Making Sense of Structural Disadvantage, Gender and Health Status. Soc Scie Med1991; 32 (4): 425-36.

29. Piccinelli M, Simon G. Gender and cross-cultural differences in somatic symptoms associated with emotional distress. An international study in primary care. Psychol Med 1997 Mar; 27(2): 433-44.

30. Gulbrandsen P, Fugelli P, Hjortdahl P. Psychosocial problems presented by patients with somatic reasons for encounter: tip of the iceberg? Fam Pract1998 Feb; 15(1): 1-8.

31. Johansson EE, Hamberg K, Westman, G, Lindgren $\mathrm{G}$. The meanings of pain: an exploration of women's descriptions of symptoms. Soc Sci Med1999 Jun; 48(12): 1791-802.

32. Wileman L. May C. Chew-Graham C. Medically unexplained symptoms and the problem of power in the primary care consultation: a quality study. Fam Prac2002; 19(2):178-82.

33. Borges S, Waitzkin H. Women's narratives in primary care medical encounters. Wom Health 1995; 23(1): $29-56$.

34. West C. Reconceptualizing gender in physicianpatient relationship. Soc Sci Med 1993; 36(1): 57 66.

35. Méndez Carlos. La Fibromialgia, los Trastornos Funcionales Digestivos y la Depresión Monografía Postgrado Reumatología. Universidad de la República Uruguaya. Cátedra de Reumatología. Febrero 2002. [citado 10 de nov. 2003]. Disponible en: www.afibrom.org/Trabajopremiado.doc.

36. Gill D, Sharpe M. Frequent consulters in general practice: a systematic review of studies of prevalence, assotiations and outcome. J Psychosm Research 1999; 47(2):115-30.

37. Ruiz- Doblado S, Pérez A, Lara L. Identificación de síntomas depresivos en atención primaria (I): Factores relacionados. Anal Psiquiatr 1997; 13(4): 139-48.

38. Alijotas J, Alegre J, Fernández-Solá J, Clots J, et al. Documento de consenso sobre el diagnóstico del síndrome de fatiga crónica en Catalunya. Med Clin 2002; 118: 73-6.

39. Uceda J, González I, Fernández C, Hernández R. Fibromialgia. Rev Esp Reumatol 2000; 27(10): 414-6.
40. Sanz-Carrillo C, Arévalo E, García -Campayo J. Somatización y familia: intervención del médico de atención primaria. Aten Prim 1998; 21(8): 545-52.

41. Moreno I, Montaño A. Fibromialgia. Rev Esp Reumatol 2000; 27(10): 436-41.

42. Claraco LM, Monreal A, García J. Manejo del paciente somatizador en atención primaria. Form Méd Cont 1999; 6(8): 536- 43.

43. Caballero L. Somatizaciones y síndromes somáticos funcionales. ¿Galgos o podencos?. Semergen 2002; 28(4): 175-6.

44. Arnetz BB. Causes of change in the health of populations: a biopsychosocial viewpoint. Soc Sci Med 1996; 43(5): 605-8.

45. García Campayo J, Sanz Carrillo C. Eficacia de la psicoterapia analítica grupal en enfermos somatizadores. Actas Esp Psiquiatr2000; 28(2): 105-14.

46. Iglesias C, Oller M. Manejo del paciente con trastornos de somatización. Jano 2000; 58(1326): 45-51.

47. López Herrero LS. La histeria cien años después. Aten Prim 1997; 19(3):151-3.

48. Aparicio D. Dolor: el interlocutor determina el destino del síntoma. Mujer y Salud 2002; 10:17-8.

49. Luis de Arribas LM, Martínez FJ. Lo psicosomático en atención primaria. Aten Prim 2000; 25(9): 112-8.

50. Tizón JL. La atención primaria a la salud mental: una concreción de la atención sanitaria centrada en el consultante. Aten Prim 2000; 26(2):101-17.

51. Richman AJ, Jason AL. Gender biases underlaying the social construction of illness states: the cases of chronic fatigue syndrome. Current Sociology 2001 May; 49(3):15-29.

52. Valls C. Diagnóstico diferencial del dolor de las mujeres. Mujer y Salud 2002; 10: 4-8.

53. Stoppard JM. A Feminist Position on Mental Health. Sex Roles 1987 June11-12; 16: 669-71.

54. Organización Mundial de la Salud. Gender and women`s mental health. 2002 [citado 1 de ene. 2002] Disponible en: http://www.who.int/mental_health/prevention/genderwomen/en/.

55. Romito P. Work and Health in Mothers of Young Children. Int J Health Serv 1994; 24 (4): 607-28. 
56. Hankin JR. Gender and Mental Illness. Resea Com Mental Health 1990;6: 183-201.

57. Annandale E, Hunt K . Masculinity, Feminity and Sex: An Exploration of Their Relative Contribution to Explaining Gender Differences in Health. Soc Health Illness 1990; 12 (1): 24-46.

58. Wool CA, Barsky AJ. Do women somatize more than men? Gender differences in somatization. Psychosomatics 1994Sep; 35(5): 445-52.

59. Stewart DE. Women's health and psychosomatic medicine. J Psychosom Research 1996 Mar;40(3): 221-6.

60. Gijsberg Van Wijk CMT, Van Vliet KP, Kolk AM Gender perspectives and quality of care: towards appropriate and adequate health care for women. Soc Sci Med 1996;43(5):707-20.

61. Busfield J. Mental Illness as Social Product or Social Construct: A Contradiction in Feminists' Arguments? Soc Health Illness 1988 Dec; 10:521-42.

62. Malterud K, Okkes I. Gender differences in general practice consultations: methodological challenges in epidemiologycal research. Fam Pract 1998 Oct 15(5):404-10.
63. Malterud K. Symptoms as a source of medical knowledge: understanding medically unexplained disorders in women. Fam Med 2000 Oct; 32(9): 603-11.

64. Tubert S. Construcción cultural de la feminidad. En: Salud mental y género. Aspectos psicosociales diferenciales en la salud de las mujeres. Madrid: Instituto de la Mujer. Ministerio de Trabajo y Asuntos Sociales;2000.

65. Bayo-Borrás R. Cuando no hay palabras para decirlo, hay cuerpo para expresarlo. Mujer y Salud 2002;10:14-6.

66. Delgado A. Salud y género en las consultas de atención primaria. Aten Prim2001 Feb;27 (2):75-8.

67. Tubert S. Psicopatología femenina y malestar en la cultura. Symposium Internacional Mujer y Calidad de Vida, CAPS, Barcelona;1990.

68. Van Den Brink-Muinen A. Women`s health care: for won and Hig? Soc Sci Med; 44(10):1541-51.

69. Rodríguez R (ed.) El malestar silenciado. La otra salud mental. Madrid: Isis Internacional. Ediciones de las Mujeres 14;1990. 